\title{
Medical Image of the Week: Bronchus Sui
}
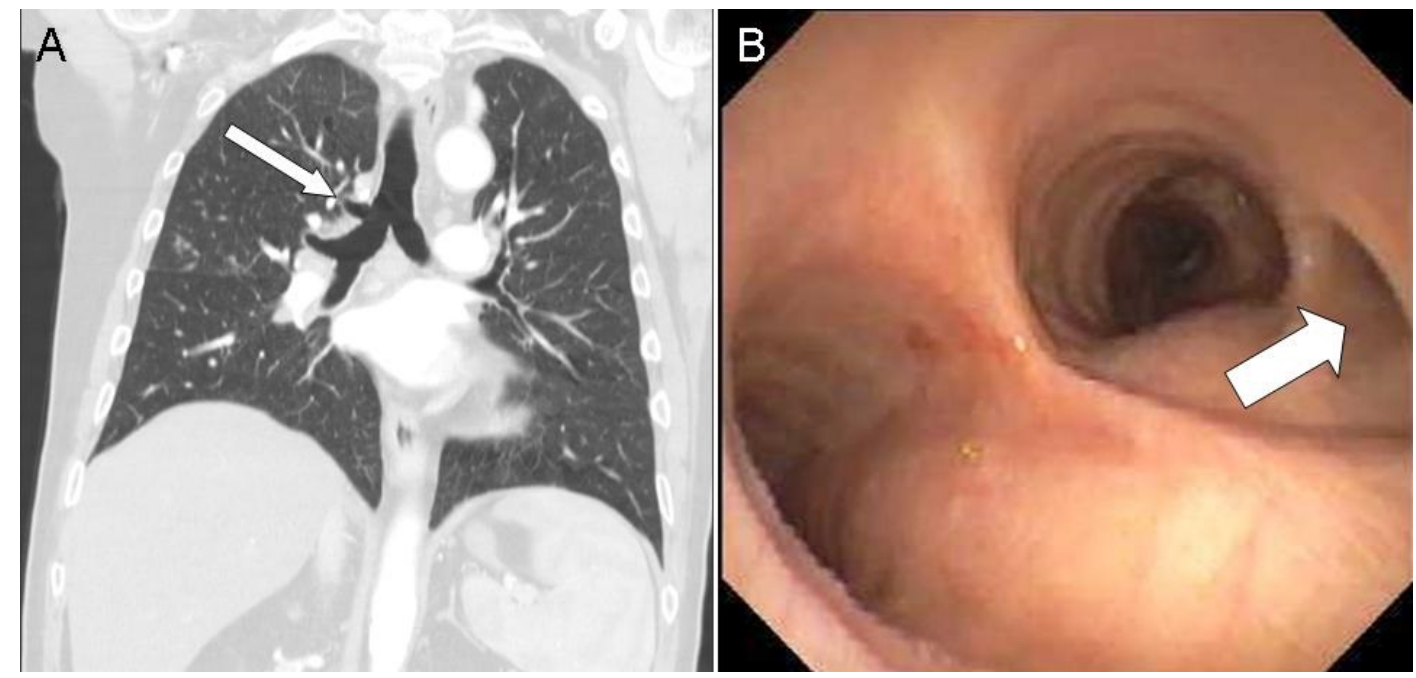

Figure 1. Panel A: Coronal view of the thoracic CT scan showing the right upper lobe superior subsegment bronchus taking off from the trachea (arrow). Panel B:

Bronchoscopy confirming the tracheal origin of the superior subsegment bronchus.

A 65 year-old man presented to the outpatient clinic for evaluation of a chronic cough. The patient underwent CT Chest (Figure 1A) that shows a right upper lobe (RUL) infiltrate with an incidental right-sided tracheal bronchus. The incidence of right-sided tracheal bronchus is $0.1-2 \%$ and has different subtypes. This patient has the most common subtype called a displaced tracheal bronchus as the aberrant, superior segment has normal RUL branching, which coexists with normal right-sided anatomy except that the one branch of the upper lobe bronchus is missing. A true "bronchus sui" (pig bronchus) is when the RUL takes off from the trachea and the right main bronchus acts as the bronchus intermedius feeding the right middle and lower lobes. A tracheal bronchus is usually asymptomatic but can be associated with recurrent pneumonia, chronic bronchitis and bronchiectasis. Bronchoscopy (Figure 1B) was performed for the purpose of RUL bronchoalveolar lavage and endobronchial ultrasound of the mediastinal lymphadenopathy.

Nathaniel Reyes MD, Bhupinder Natt MD, Janet Campion MD Division of Pulmonary and Critical Care Medicine Arizona Respiratory Center University of Arizona

Tucson, AZ

\section{Reference}

Findik S. Tracheal bronchus in the adult population. J Bronchology Interv Pulmonol. 2011;18(2):149-52. [CrossRef] [PubMed] 